associated with tearing. The corneal epithelium may be injured but recovery occurs within 48 hours. $^{2}$

Three of our patients complained most about the initial irritation caused by the correction fluid but suffered no decrease in visual acuity. Their symptoms settled quickly. However, the fourth patient experienced a marked reduction of visual acuity in the right eye which took a week to recover. In the above cases, a significant proportion of the correction fluid had come off the cornea at 1 week. Thus a conservative approach proved effective in these school children.

Besides being packaged as a liquid, correction fluid is also available in the form of a rollerball pen and as transfer sheets. These latter products are much less likely to lead to ocular injury, particularly when used by young children, making them more suitable for school use.

\section{J. McLean}

Department of Ophthalmology

The North Middlesex Hospital

Sterling Way

London N18 1QX

UK

Correspondence to:

C. J. McLean

Moorfields Eye Hospital

City Road

London EC1V 2PD

UK

\section{References}

1. Pointer J. Typewriter correction fluid: a new substance of abuse. J Toxicol Clin Toxicol 1982;19:493-9.

2. Zagora E. Halogenated compounds. In: Eye injuries. Florida: CC Thomas, 1970:359.

Sir,

A Case of Conjunctival Involvement with Mycosis Fungoides Successfully Treated by Surgery

Ocular localisation of mycosis fungoides may occur in up to a third of advanced cases and most frequently presents as tumours or infiltration of the eyelids. ${ }^{1,2}$ However, conjunctival involvement with mycosis fungoides has been reported only rarely. ${ }^{1,3}$

Here we report a case of mycosis fungoides involving the conjunctiva, successfully treated by surgery.

\section{Case Report}

The patient was a 34-year-old man with a 7-year history of cutaneous T-cell lymphoma stage IIb (TMN classification: T2, N1, M0). The disease was initially controlled with PUVA therapy. In 1990, however, the sudden spread of the lymphoma led to treatment with recombinant interferon- $\alpha 2 \mathrm{a}$ (rINF-

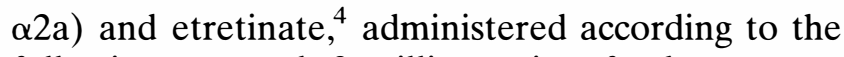
following protocol: 3 million units of subcutaneous rINF- $\alpha 2$ a every other day plus $50 \mathrm{mg} /$ day etretinate. This treatment reduced the skin lesions by approximately $70 \%$. The drugs were therefore tapered to a maintenance dose of $25 \mathrm{mg}$ etretinate twice a week from January 1992. Treatment was suspended in September 1993 because the skin manifestations had completely regressed.

During a check-up at the beginning of 1994, signs of relapse of the disease were noted and the patient reported a conjunctival lesion in the left eye. Dermatological examination revealed two new infiltrating plaques on the abdomen. Ophthalmological examination showed a nodule involving the temporal region of the eyeball about $2 \mathrm{~mm}$ from the corneal limbus and touching the external canthus of the left eye (Fig. 1). The conjunctiva seemed to have thickened around the lesion. The nodule had a rubbery consistency and there were many telangiectatic vessels on its surface. Slit lamp observation showed that the nodule was completely mobile with respect to the sclera when touched with cotton under local anaesthetic. It was decided to remove the lesion surgically for both therapeutic and diagnostic purposes. Bupivacaine-adrenaline (Marcaina with adrenalin R) was injected under the conjunctiva inferior to the nodule and cauterisation of all the perilesional conjunctiva was performed. The conjunctival tissue inside the cauterisation was completely excised. Despite the fact that the lesion seemed to be completely isolated from the underlying layers, the corresponding episclera was also excised. To facilitate cicatrisation an advancement of a conjunctival flap was performed.

Sections of the nodule were stained with haematoxylin-eosin and periodic acid-Schiff (PAS) and examined by light microscopy. Sheets of mediumsized atypical lymphoid cells involving the subepithelial stroma were observed (Fig. 2). Pautrier microabscesses were also noticed in the epithelium. At

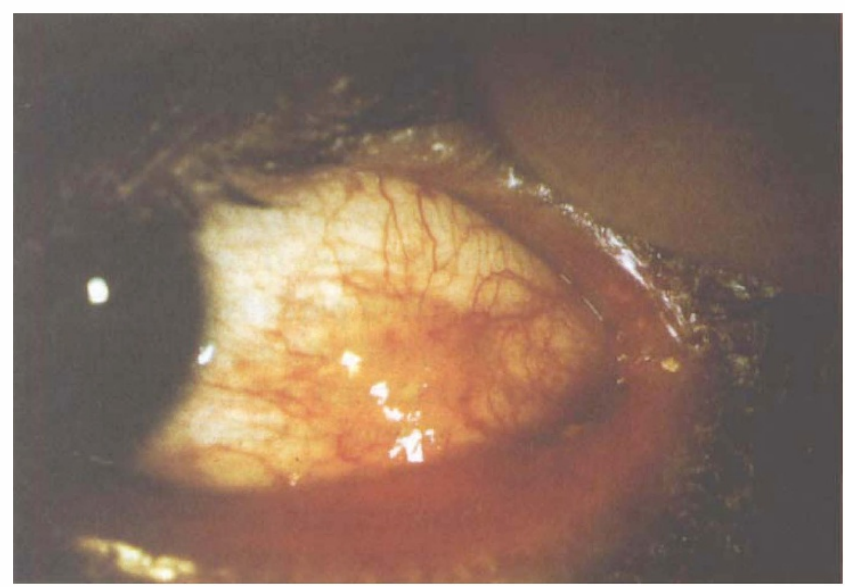

Fig. 1. Clinical appearance of the conjunctival nodule. 


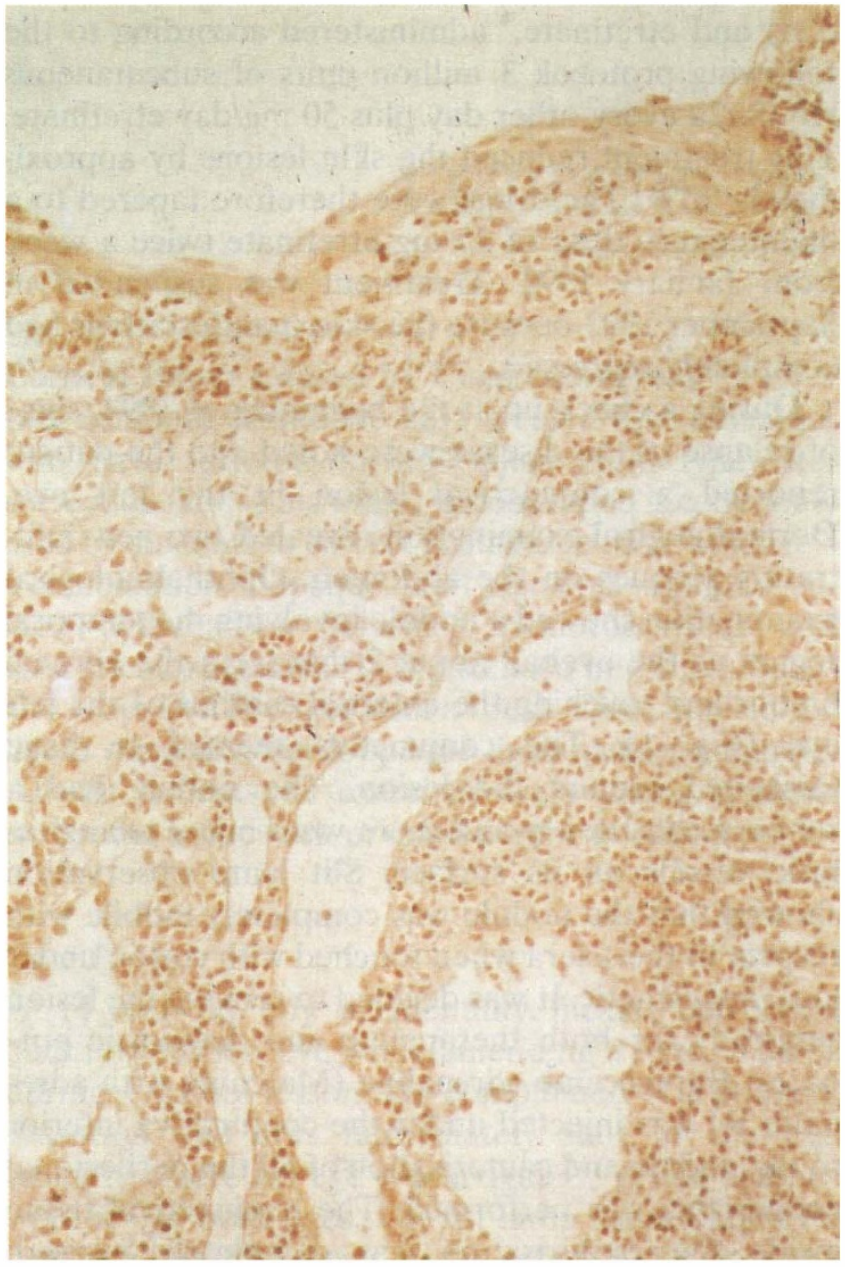

Fig. 2. Sheets of medium-sized atypical lymphoid cells involving the dermal stroma and Pautrier microabscesses in the epithelium (haematoxylin-eosin, $\times 15$ ).

higher magnification, some of these lymphocytes showed large convoluted nuclei. Immunohistochemical study of the tissue showed that the infiltrating lymphocytes were of T-cell origin $\left(\mathrm{CD}^{+}\right.$and $\left.\mathrm{CD}^{+}\right)$.

These data led to the diagnosis of conjunctival localisation with mycosis fungoides. Therapy with low doses of rINF- $\alpha 2$ a $(3$ million units 3 times a week) and $25 \mathrm{mg}$ etretinate 3 times a week was reinstated and led to a gradual regression of cutaneous plaques. A year later, the patient is still on this therapy and no eye or skin relapses have occurred.

\section{Comment}

Eye anomalies are common in patients with mycosis fungoides. ${ }^{2}$ The most frequent are seborrhoeic blepharoconjunctivitis, cicatricial ectropion, diffuse punctate epithelial defects, conjunctivitis and meibomianitis. However, it is rare to observe a primitive localisation of mycosis fungoides in the eye..$^{1,2,5}$ In a review on the subject, ${ }^{1}$ concerned with 36 consecutive mycosis fungoides patients with histologically documented eye involvement, the authors reported that the eyelids were the most commonly affected region and only six cases had a direct conjunctival localisation. Unfortunately the authors did not report how these lesions were treated. In another report of conjunctival involvement with mycosis fungoides, O'Day et $_{\mathrm{al}}{ }^{3}$ suggested that superficial X-ray therapy can be used to treat these conjunctival localisations of mycosis fungoides, although the authors recognised that side effects such as cataract formation should be anticipated.

We report this case because of its rarity and because of the excellent results obtained with surgery and low doses of rINF- $\alpha 2$ a plus etretinate. When possible, this treatment is preferable to X-ray therapy, being just as efficacious and without significant side-effects for the eye.

Pietro Rubegni ${ }^{1}$

Maria Laura Flori ${ }^{1}$

Vincenzo Mittica ${ }^{2}$

Gian Marco Tosi ${ }^{2}$

Michele Fimiani ${ }^{1}$

${ }^{1}$ Department of Dermatology

${ }^{2}$ Department of Ophthalmology

University of Siena, Italy

Correspondence to:

Dott. Pietro Rubegni

Department of Dermatology

University of Siena

Policlinico 'Le Scotte'

I-53100 Siena, Italy

Tel: (+39) 577-40190

Fax: $(+39) 577-44238$

\section{References}

1. Stenson S, Ramsay DL. Ocular findings in mycosis fungoides. Arch Ophthalmol 1981;99:272-7.

2. Leib ML, Lester H, Braunstein RE, Edelson RL. Ocular findings in cutaneous T-cell lymphoma. Ann Ophthalmol 1991;23:182-6.

3. O'Day J, Rostein H, Weiner JM. Conjunctival involvement with mycosis fungoides in a patient receiving PUVA therapy. Ophthalmology 1985;92:109-13.

4. Therstrup-Pedersen K, Hammer R, Kaltoft K, Soogard $\mathrm{H}$, Zachariae $\mathrm{H}$. Treatment of mycosis fungoides with recombinant interferon-alpha $2 \mathrm{a}$ alone and in combination with etretinate. Br J Dermatol 1988;118:811-8.

5. Vogiatzis KV. Lymphoid tumors of the orbit and ocular adnexa: a long term follow-up. Ann Ophthalmol 1984;16:1046-55.

Sir,

\section{Hormonal Treatment of Bilateral Optic Nerve Meningioma}

Bilateral optic nerve sheath (ONS) meningiomas are rare. To date, of the approximately 500 cases of primary ONS meningiomas reported, ${ }^{1}$ only $5 \%$ have been bilateral. Neurofibromatosis type II is associated with ONS meningioma and a case of bilateral 